\title{
Un modelo econométrico de la rentabilidad: Caso CMAC Arequipa. 2002-20161
}

\section{An econometric model of the profitability: CMAC Arequipa Case. 2002-2016}

\author{
Edwin Alberto Bazán Díaz ${ }^{2}$ \\ Universidad Nacional Mayor de San Marcos \\ edwin.bazan@unmsm.edu.pe \\ https://orcid.org/0000-0002-4415-6539
}

Recibido: 28/10/2019 - Aceptado: 21/05/2020 - Publicado: 18/08/2020

\section{RESUMEN}

El presente trabajo de investigación tiene como objetivo, conocer el impacto de las variables: los créditos directos, la tasa de inflación y la variación anual del PBI real expresado como una variable dummy, sobre la rentabilidad (ROA y ROE) de la Caja Municipal de Ahorro y Crédito Arequipa durante el período 2002-2016. Para ello se ha visto conveniente tomar la información estadística de fuentes secundarias. De manera global ambos modelos son significativos; pero, el Durbin-Watson del ROA es más alto respecto al del ROE, aproximándose al valor de 2. Al aplicar el modelo econométrico con un rezago en términos de determinantes específicos de la CMAC Arequipa de la rentabilidad, el retorno sobre los activos (ROA) y el retorno sobre el patrimonio (ROE), los créditos directos son significativos y a la vez tienen un efecto negativo en la rentabilidad para ambos.

Palabras clave: Rentabilidad; microfinanzas; créditos directos; riesgo; crecimiento; gestión.

JEL: B30, C30, G21.

(C) Los autores. Este artículo es publicado por Pensamiento Crítico de la Facultad de Ciencias Económicas, Universidad Nacional Mayor de San Marcos. Este es un artículo de acceso abierto, distribuido bajo los términos de la licencia Creative Commons Atribucion - No Comercia_Compartir Igual 4.0 Internacional. (http://creativecommons.org/licenses/by-nc-sa/4.0/) que permite el uso no comercial, distribución y reproducción en cualquier medio, siempre que la obra original sea debidamente citada. 


\section{ABSTRAC}

The present research work aims to know the impact of variables: direct credits, the inflation rate and the annual variation of real PBI expressed as a dummy variable, on profitability (ROA y ROE) of the CMAC: Arequipa during the period 2002-2016. For this inconvenient, we will get the statistic information from secondary sources. Globally, both models are significant; but, the Durbin-Watson of the ROA is highest as regards the of the ROE approaching the value of 2 . By applying the econometric model with a lag in terms of specific determinants of the CMAC Arequipa profitability; return on assets (ROA) and return on heritage (ROE), direct credits are significant and at the same time have a negative effect on profitability for both.

Keywords: Profitability; microfinance; direct credits; risk; growth; management. JEL: B30, C30, G21. 


\section{Introducción}

El surgimiento de las cajas municipales para Jaramillo, M. (2014, p. 3) "datan de inicios de los años ochenta. La experiencia previa con préstamos pequeños para grupos socioeconómicos bajos y medianos era variada y contemplaba programas de préstamo y préstamos colectivos, tanto en ciudades como en zonas rurales".

Las Cajas Municipales de Ahorro y Crédito (CMAC) han logrado a lo largo de sus 30 años de creación, posicionarse como las entidades expertas en el sector de las microfinanzas, trabajando por el desarrollo de los micros y pequeñas empresas (MYPES), así como lograr la inclusión de peruanos de escasos recursos.

En general se debe mencionar que la CMAC Arequipa direcciona sus actividades financieras, de ahorros y de créditos directos a todos los sectores económicos. Según Estadísticas de la Superintendencia de Banca y Seguros (2016) la cartera de créditos directos tiene un mayor énfasis a las pequeñas empresas con S/ 1,478'039,000 y a las micro empresas de S/ 862'379,000.

Los créditos a los diferentes estratos económicos se orientan mayormente, en primer lugar, al sector comercio, en segundo lugar, a transporte y en tercer lugar a actividades inmobiliarias, empresariales y de alquiler al 2016.

Dentro de este contexto, el objetivo del presente artículo consiste en realizar una evaluación cuantitativa expresado en dos modelos econométricos que permiten medir la rentabilidad (ROA y ROE) de la Caja Municipal de Ahorro y Crédito: Arequipa durante el período 2002-2016, considerando como variables: los créditos directos (vigente, refinanciada, reestructurada, vencida, en cobranza judicial), la inflación y la variación del PBI real expresada con variable dummy.

\section{Metodología}

De tal manera, para la actual investigación esta es de tipo explicativa porque se busca obtener la medición, contribución, influencia y de qué mane- 
ra afecta las variables independientes en las variables dependientes ROA y ROE y responder a la hipótesis general como a las hipótesis específicas.

Esta investigación es aplicada, porque se trata de elegir el mejor modelo econométrico de los dos que explique estadísticamente un valor de Durbin - Watson aproximadamente a 2, donde no exista autocorrelación, por la influencia de los créditos directos, la inflación y la variación del PBI real expresada con variable dummy sobre el ROA y ROE.

Se desea conocer el nivel de investigación, el cual es descriptivo, porque se explica los hechos económicos-financieros desde el 2002 hasta el 2016, alrededor de la CMAC Arequipa considerando ciertos aspectos macroeconómicos y créditos directos.

La metodología que se aplica es mixta: porque se utiliza un componente cuantitativo y un componente cualitativo, se toma en cuenta las variaciones de año a año, entonces se asume 1 por un incremento de la tasa de crecimiento del PBI real y 0 cuando hay una disminución del PBI real.

Y finalmente presenta el diseño: Descriptivo - correlacional. En lo que concierne a correlacional es por se trata de hallar el grado de relación entre las variables en estudio y también se establece que es no experimental.

Se empleará el enfoque mixto que es un proceso que recolecta, analiza, vincula datos cuantitativos y cualitativos en este mismo estudio y se ha tomado información secundaria mostrada por diversas instituciones como: SBS, BCR, INEI. La información de los créditos directos es anual y son el resultado del promedio de 12 meses.

Una opinión importante es la entrevista otorgada por el señor gerente de la CMAC Arequipa, Agencia Mendiola: Elvis Espejo Rivadeneyra, quién manifiesta la solicitud de crédito de algunas personas sin ninguna garantía prendaria.

Se ha analizado la evolución de ciertas variables financieras, el comportamiento gráfico del ROA y el ROE y de ciertas variables macroeconómicas través de los 15 años del período en estudio.

Sobre el uso de las variables del sector real y financiero quiero considerar la apreciación de Aguilar (2013, p. 162): 
Los estudios sobre crecimiento y desarrollo financiero proporcionado por Levine (2005) discuten la falta de un indicador idóneo que sea capaz de dar cuenta de los distintos mecanismos a través de los cuales el desarrollo de las finanzas puede afectar la actividad real. King y Levine (1993) mencionan que la práctica común es trabajar con el "tamaño" del sector financiero en relación a la actividad económica medido ya sea como el total de créditos o depósitos del sistema como porcentaje del PBI. Esta medida se conoce como profundidad financiera.

Se ha realizado un análisis correlacional de los créditos directos, la inflación y la variación del PBI real expresada en valores reales respecto al ROA y al ROE utilizando el Software de Microsoft Excel.

Se va a utilizar un modelo de regresión lineal múltiple - para cuyos resultados se usa el software Econometric EViews 9- que utiliza una variable financiera: los créditos directos y las variables macroeconómicas como: la inflación y la variación del PBI real expresada como una variable dummy.

$$
\begin{aligned}
& \mathrm{ROA}_{\mathrm{t}}=\beta_{0}+\beta_{1} \mathrm{X}_{\mathrm{t} 1}+\beta_{2} \mathrm{X}_{\mathrm{t} 2}+\beta_{3} \mathrm{~d}_{\mathrm{t} 3}+\mathrm{u}_{\mathrm{t}}(1) \\
& \mathrm{ROE}_{\mathrm{t}}=\beta_{0}+\beta_{1} \mathrm{X}_{\mathrm{t} 1}+\beta_{2} \mathrm{X}_{\mathrm{t} 2}+\beta_{3} \mathrm{~d}_{\mathrm{t} 3}+\mathrm{u}_{\mathrm{t}}(2)
\end{aligned}
$$

Donde:

$\mathrm{X}_{1=}$ Los créditos directos de la caja municipal: Caso CMAC Arequipa durante el período 2002-2016.

$\mathrm{X}_{2=}$ La tasa de inflación anual en el Perú en el tiempo, durante el período 2002-2016.

$\mathrm{d}_{3=}$ Las variaciones del PBI real anual en el tiempo, medido como variable dicotómica es decir la variable dummy, durante el período 20022016.

Y= La Rentabilidad de la caja municipal (ROA, ROE): Caso CMAC Arequipa durante el período 2002-2016.

$\beta_{0}=$ Corresponde al valor autónomo del ROE, es el comportamiento de la rentabilidad, sin efecto de los créditos directos, inflación y la variación del PBI real. 
$\beta_{1}=$ Corresponde al valor del comportamiento de la rentabilidad respecto al efecto de los créditos directos.

$\beta_{2}=$ Corresponde al valor del comportamiento de la rentabilidad respecto al efecto de la tasa de Inflación.

$\beta_{3}=$ Corresponde al valor del comportamiento de la rentabilidad respecto al efecto de la variación anual del PBI real.

$u_{t}=$ Es el efecto de los errores aleatorios.

\section{Marco Teórico}

Con el DS № 007-2008-TR se establece el texto único ordenado de la ley de promoción de la competitividad, formalización y desarrollo de la empresa y del acceso al empleo decente. LEY MYPE. En la tabla 1 se expresan algunos alcances respecto a esta Ley:

Tabla 1

Características de las MYPES

\begin{tabular}{|l|l|}
\hline \multicolumn{1}{|c|}{ MICROEMPRESA } & \multicolumn{1}{c|}{ PEQUEÑA EMPRESA } \\
\hline $\begin{array}{l}\text { Los niveles de venta anuales: hasta el monto máximo } \\
\text { de 150 UIT. }\end{array}$ & $\begin{array}{l}\text { Los niveles de venta anuales: hasta el monto desde 150 } \\
\text { UIT hasta 1700 UIT. }\end{array}$ \\
\hline $\begin{array}{l}\text { Número de trabajadores: de uno (1) hasta diez (10) } \\
\text { trabajadores inclusive. }\end{array}$ & $\begin{array}{l}\text { Número de trabajadores: de uno (1) hasta cien (100) } \\
\text { trabajadores inclusive. }\end{array}$ \\
\hline
\end{tabular}

Fuente: Artículo 5 del Título I del DS № 007-2008-TR

Elaboración propia

Desde siempre se ha manifestado el vínculo entre el sector financiero y el sector real de la economía. Al respecto se menciona una serie de planteamientos teóricos y un trabajo empírico.

a. Entre los planteamientos teóricos se mencionan:

Keynes (1981, p. 32) referente a la ganancia en su libro de Teoría General de la Ocupación, el interés y el dinero indica "el excedente de valor que da la producción resultante sobre la suma del costo de factores y el costo de uso es la ganancia, o, como lo llamaremos, el ingreso del empresario". 
Y en cuanto al costo de dinero de un préstamo Keynes (1981, p. 199) manifiesta "la tasa monetaria de interés no es otra cosa que el porciento de excedente de una suma de dinero contratada para entrega futura". En ambos párrafos se observa ya la relación entre ambos sectores".

Entonces desde el punto de vista del ahorrista Markowitz (1952, p. 78) argumenta "si dos o más precios tienen el mismo valor entonces estos o la combinación de estos son buenos como la combinación de otra cualquiera"

Acerca de una empresa, Ross, Westerfield y Jordan (2014, p. 493) afirman "Modigliani y Miller (MM o M \& M) argumentaban respecto a los accionistas de una empresa que el valor de la empresa siempre es el mismo en diferentes estructuras de capital. Ninguna estructura de capital es mejor o peor que cualquier otra".

En cuanto a la corriente austriaca, Schumpeter (1976, p. 82) menciona "el crédito es necesario para la creación de nuevas combinaciones (el distinto uso de factores productivos que da el empresario a los ya existentes), fue necesario en su momento para la creación de antiguas empresas y que también se utiliza en viejas combinaciones".

Para Schumpeter (1976, p. 115) el accionar económico puede expresarse en el siguiente aspecto "el crédito es esencialmente la creación de poder de compra con el propósito de transferirlo al empresario (...). Solamente así puede surgir el desenvolvimiento económico de la simple corriente circular, en situación de equilibrio perfecto".

Duflo y otros (2013, p. 1) en el artículo The miracle of microfinance? Evidence from a randomized evaluation, argumentan "El microcrédito ha generado un entusiasmo y una esperanza considerables para el alivio rápido de la pobreza. En 2006, Mohammad Yunus y el Grameen Bank recibieron el Premio Nobel de la Paz, por su contribución a la reducción de la pobreza mundial".

Al respecto, Duflo y otros (2013) argumentaron en contraste con las afirmaciones que algunas veces hacen las IMF y otras, la demanda de micropréstamos está lejos de ser universal. Al final de nuestro período de estudio de tres años, solo el 38\% de los hogares toma prestado de una IMF. 
Después de reflexionar sobre los diferentes enfoques económicos, se consideró que la teoría Schumpeteriana es aquella que se ajusta mejor a la problemática de la presente investigación.

Dado nuestro enfoque hacia las microfinanzas, los lineamientos del pensamiento Schumpeteriano relacionados hacia el empresario, crédito y comportamiento social son los más acertados para adentrarnos más en el desenvolvimiento económico y el microcrédito.

b. En lo que respecta a trabajos empíricos se menciona uno muy importante:

Previamente expongo a uno de los economistas con la preocupación de medir las cuentas nacionales y otras mediciones económicas y Castilla (2015, p. 437) indica "Lawrence R. Klein, premio nobel de economía en 1980 argumentaba que, en los años 60, 70 y 80 del siglo pasado se crea la industria de la predicción económica para todos los países y para el mundo con modelos econométricos. Lo mismo opinaba respecto a la aparición de una parte nueva de la economía: la economía financiera".

Y con este párrafo queda el criterio de nuestro trabajo respecto al uso de nuestras variables, Castilla (2015, p. 444) asevera "todo ello significa que la cuantificación de variables empresariales relacionadas con las finanzas y otras actividades (...) es el uso abierto de la 'econometríá en dichas actividades empresariales".

En el documento "Análisis de los Factores que influyen en la rentabilidad de las cajas municipales de Ahorro y crédito en el Perú", la autora Bayona, (2013) analiza mediante la representación econométrica de panel de datos dinámicos el estudio a un conjunto de datos: rubros financieros, número de personal y el crecimiento del Producto Bruto Interno.

Bayona, (2013, p. 86) concluye para el ROE y el ROA, que las variables analizadas como: El volumen de actividad, el apalancamiento, los ingresos por servicios financieros (INGSF) y el crecimiento del PBI, presentan impactos opuestos en los resultados de la estimación. A pesar de que las variables son más significativas no son válidas por la presencia de autocorrelación, sobreidentificación que 
existe entre las variables en el momento de estimar teniendo como variable dependiente el ROA. Entonces sería recomendable un modelo con presencia de un componente autorregresivo, que permite levantar la sobreidentificación que existe entre las variables en el modelo de regresión.

\subsection{Riesgo}

Respecto al riesgo (Conde, p. 81) manifiesta que: Entonces hay que subrayar que asumir riesgos es algo inherente al ejercicio de la actividad financiera y a la obtención de resultados positivos o negativos. Administrar el riesgo es establecer una gestión compuesta del resultado esencial para la sustentabilidad en el largo plazo de las instituciones microfinancieras y así incorporar en la práctica este concepto como uno de los más grandes desafíos económico-financiero actualmente.

La Caja Arequipa ha demostrado que es posible financiar con un alto riesgo y relativo éxito proyectos como el caso de una señora que tiene su carretilla y vende huevos de codorniz.

Para atenuar el riesgo un elemento a tomar en cuenta es el Credit Scoring que permite valorar de forma automática el riesgo asociado a cada solicitud del crédito. (Puertas, R., Martí, M., 2012, p.304).

Sin embargo, la Caja Arequipa utilizó software como el Sorfy y Bantotal con el propósito de detectar la mora lo más oportuno posible.

\subsection{La Caja Arequipa y su entorno económico}

La Caja Arequipa se constituyó como asociación sin fines de lucro por Resolución Municipal N 1529 del 15 de julio 1985 al amparo del Decreto Ley $N^{\circ} 23039$ otorgándose la Escritura Pública de constitución y estatuto el 12 de febrero de 1986 ante el Notario Público Dr. Gorky Oviedo Alarcón y está inscrita en los Registros Públicos de Arequipa en el rubro "A" ficha 1316, de personas jurídicas. Su único accionista desde su constitución es el Municipio Provincial de Arequipa.

Se rige por el Decreto Supremo N 157-90-EF, la Ley General del Sistema Financiero y la Ley de Sociedades. 


\subsection{Inflación}

En el documento 20 años con inflación anualizada de un digito del BCRP (2017, p. 1) manifiesta "A inicios de 1997 la inflación anualizada llegó a la tasa de un dígito, 9,4 por ciento; dejando atrás un amplio período en el que el nivel de precios crecía a ritmos anuales de dos, tres y hasta cuatro dígitos en tiempos de hiperinflación".

En el primer gobierno del presidente García, entre agosto de 1987 y agosto de 1990 se generó una hiperinflación de 3.5 millones \%, muy diferente con las dos últimas décadas, en que la inflación promedio anual disminuyó a cerca de $3 \%$ rango meta máximo, por un manejo más responsable principalmente de las cuentas macroeconómicas: monetario y fiscal.

Acaecidos los sucesos hiperinflacionarios de finales de la década de los ochenta, en el BCRP (2017) aparecen cambios drásticos en la política monetaria y entonces se otorgó autonomía constitucional al $B C R P$, con modificaciones en su ley orgánica.

En efecto el BCRP (2017, p. 2) argumenta "En el 2002 adopta el Esquema de Metas Explícitas de Inflación (MEI) por el cual fija un objetivo de inflación. Desde 2007, la meta inflacionaria es de un rango de 1 a 3 por ciento".

En la Figura 1, el BCRP (2017) menciona desde la implementación de este esquema, la inflación promedio anual ha sido de 2.9\%, dentro del rango meta, y la más baja entre los países que siguen el mismo esquema en América Latina. Además, la dolarización del crédito se redujo significativamente desde más de 80\% a inicios del año 2000 a 29\% en el 2016.

Durante el segundo gobierno de Alan García, 2006-2011, la inflación promedio se registró en $2.85 \%$. Los principales problemas que afectaron este gobierno fueron la coyuntura financiera internacional del 2008 en EEUU, que trajo una inflación importada, así como los sucesos en nuestro país producto del cambio climático que afectó el mercado agrícola interno.

Durante gobierno de Ollanta Humala, 2011-2015, se continuo con el sistema de metas explicitas, la inflación promedio se registró en 3.6\%. Los factores que explican este resultado son: el aumento de los precios de los 
alimentos derivado de los cambios climáticos que afectaron el mercado interno, el aumento de precios del mercado energético, así como también la depreciación del tipo de cambio causada por el retiro de las políticas monetarias expansivas de la FED estadounidense para hacer frente a la competitividad china.

Durante el corto mandato de Pedro Pablo Kuczynski, 2016-2017, se continuo con el sistema de metas explícitas, la inflación promedio se registró en 2.5\%. Este resultado fue producto de la recuperación del crecimiento de las economías desarrolladas, el ajuste gradual del FED debido al aumento de la tasa de empleo estadounidense y a efectos del cambio climático; fenómeno del niño costero, que inició en diciembre del 2016 y afectó la producción agrícola del norte del país causando daños materiales en la infraestructura de las viviendas y de carreteras.

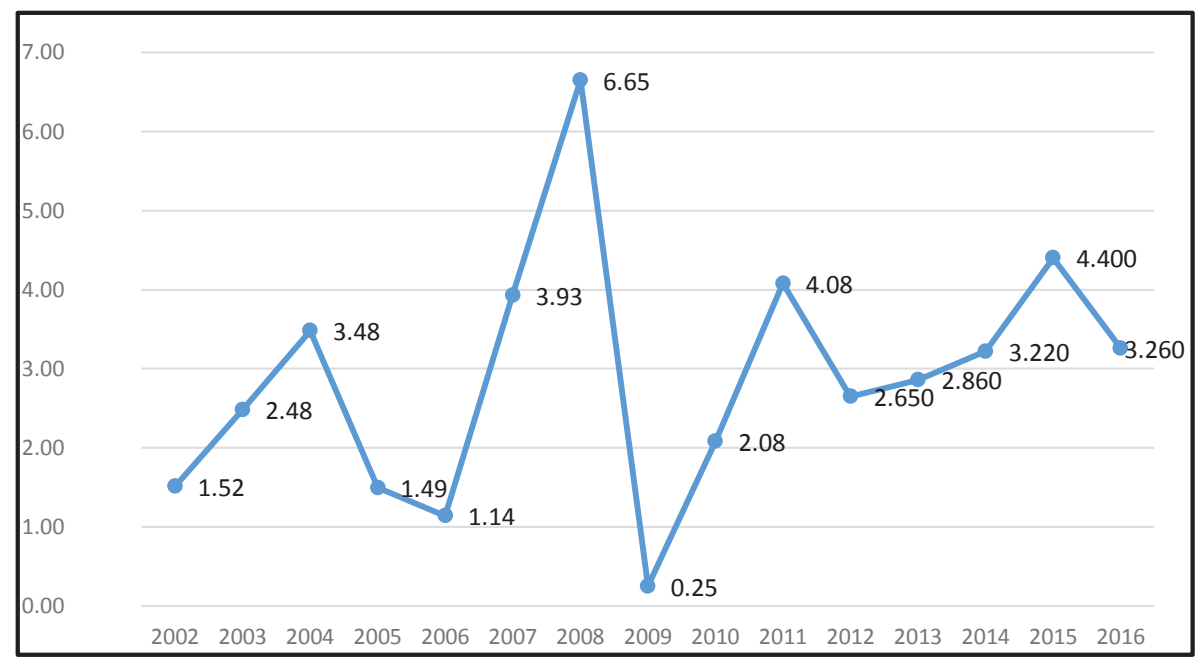

Figura 1. Evolución anual de la tasa de inflación (\%) 2002-2016 Fuente: SBS-BCR. Elaboración propia.

\subsection{Producto Bruto Interno}

Respecto al PBI, el (INEI, Metodología de Cálculo del Producto Bruto Interno Anual, p. 1) menciona "El Producto Bruto Interno se define como el valor total de los bienes y servicios generados en el territorio 
económico durante un período de tiempo, que generalmente es un año, libre de duplicaciones. El PBI, también se puede definir como el valor añadido en el proceso de producción que mide la retribución a los factores de producción que intervienen en el proceso de producción".

En la Figura 2, se observa una tendencia ascendente del PBI real o constante, en cierta medida por el aumento de los precios de los commodities, a la tasa de inflación establecida en el rango meta 1-3\%, etc. En el período 2005-2016, la economía peruana ha crecido ininterrumpidamente hasta ser el doble.

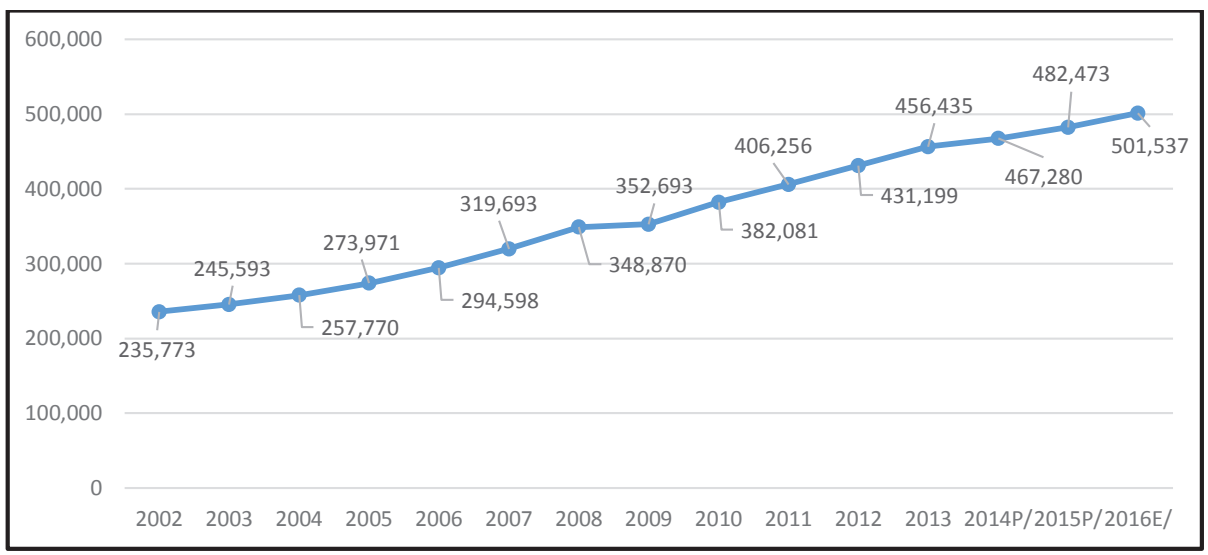

Figura 2. Evolución anual del PBI real 2002-2016 en millones de soles a precios constantes del 2007 Fuente: INEI. Elaboración propia.

En la Figura 3, se observa la tendencia del crecimiento como variación anual del PBI real, período 2002-2016. Desde el año 2002 hasta el 2008 nuestra producción más que se duplicó. En contraposición el año 2009, fue el más bajo de todos como repercusión de la crisis financiera de EEUU. Desde el 2011 viene desacelerándose el crecimiento del PBI en los sectores comercio, construcción y agropecuaio, pero sobre todo desde el 2015 en adelante, por el sector primario, basado en actividades extractivas (minería, pesca, hidrocarburos). Sobre estos datos porcentuales es que se ha establecido la variable dummy: 1 si hay incremento de un año a otro y 0 si hay un decremento de un año a otro. 


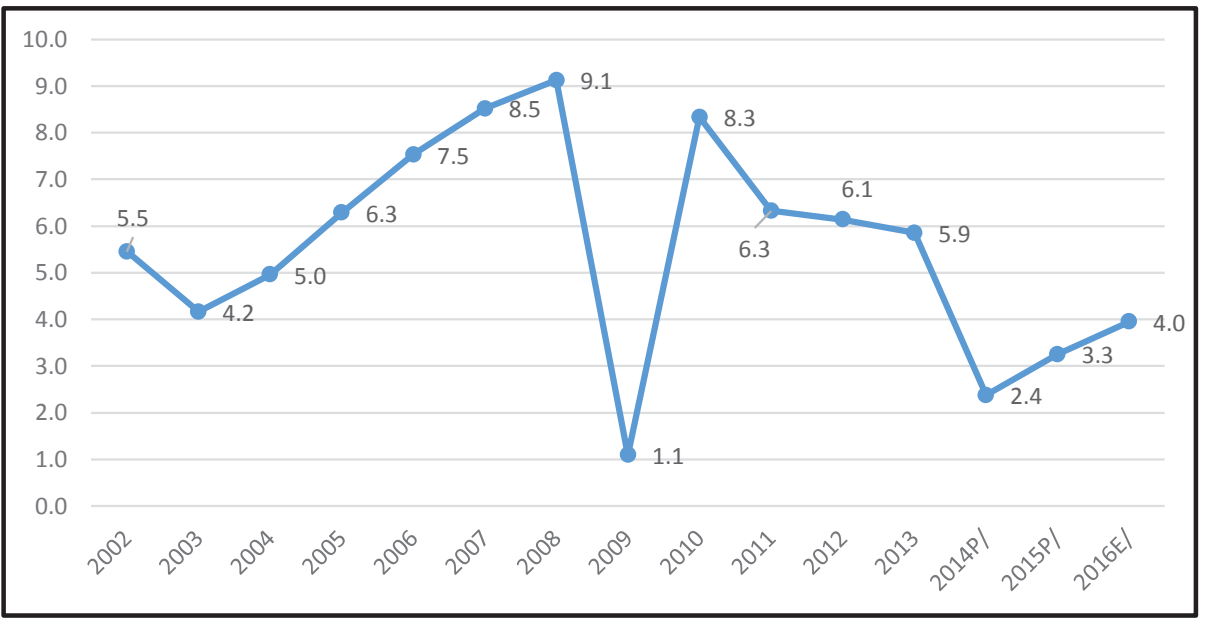

Figura 3. Evolución anual de la tasa de crecimiento del PBI real (\%) 2002-2016 Fuente: INEI. Elaboración propia.

\section{Resultados y Discusión}

\subsection{Colocaciones}

En el período del 2002 al 2013, la economía ha crecido en promedio 6.1\%. En la Figura 4, hay una tendencia exponencial de los ingresos financieros que provienen por una mayor oferta de créditos en MN. Los créditos vigentes crecen en todo el período, los créditos refinanciadosreestructurados aumentan significativamente después del 2014 y los créditos atrasados se incrementan después del 2006, expresando la situación de morosidad en aumento dado que muchas MYPES cierran sus negocios, además a partir del 2014 al 2017 el crecimiento de la economía apenas ha crecido en promedio $3 \%$.

Para el 2002 los créditos directos fueron de S/ 129 millones y de S/ 3,320 millones en el 2016, lo que representa un incremento de 2,473\%.

En la Figura 5, hay una tendencia oscilante respecto al otorgamiento de los créditos en $\mathrm{ME}$, la cual termina descendiendo por la campaña de desdolarización de las colocaciones a fin de reducir la exposición de la cartera a variaciones de tipo de cambio: los créditos vigentes tienen un comportamiento variable: crecen hasta el año 2008, los años 2009 y 2010 
caen y del 2011 crecen hasta el 2014 para luego descender en el período en estudio, los créditos refinanciados-reestructurados se han mantenido relativamente estable, para a partir del año 2014 logran tener una tendencia ascendente y finalmente los créditos atrasados tienen una tendencia ascendente hasta el año 2009 y en los siguientes cuatro años han tenido mucha cautela en el manejo para disminuirlos hasta el $50 \%$ y luego terminar ascendiendo al término del período.

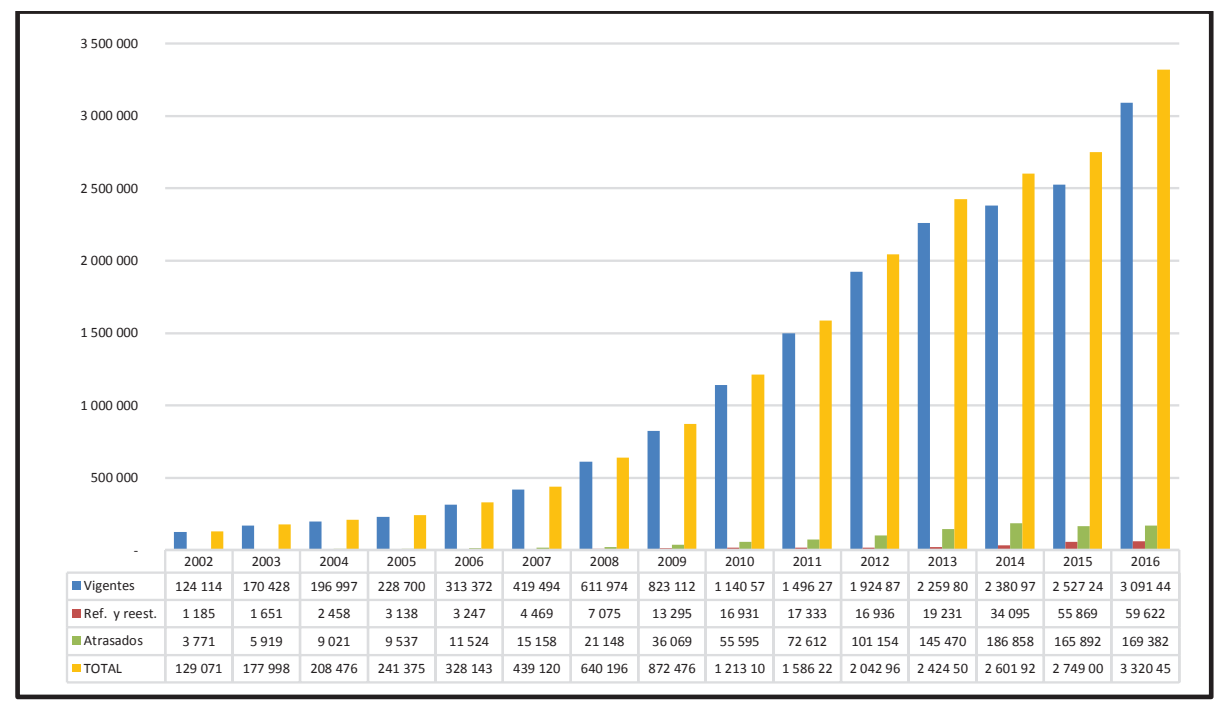

Figura 4. Promedio anual de los créditos vigentes, refinanciados -reestructurados y atrasados en MN de la CMAC Arequipa en el 2002-2016 (en miles de soles) Fuente: INDICADORES FINANCIEROS. SBS, 2002-2016

Elaboración propia

Respecto a la figura inferior, el 2002 los créditos directos fueron de S/ 70 millones y de S/ 139 millones en el 2016, lo que representa un incremento de $98 \%$.

En la Figura 6, se expresa la evolución de los créditos por sector que se colocó durante el período en estudio.

La CMAC Arequipa inicia sus operaciones con el crédito pignoraticio o prendario, el cual tiene actualmente mucha aceptación por su facilidad de acceso (http://www.cajaarequipa.pe). 


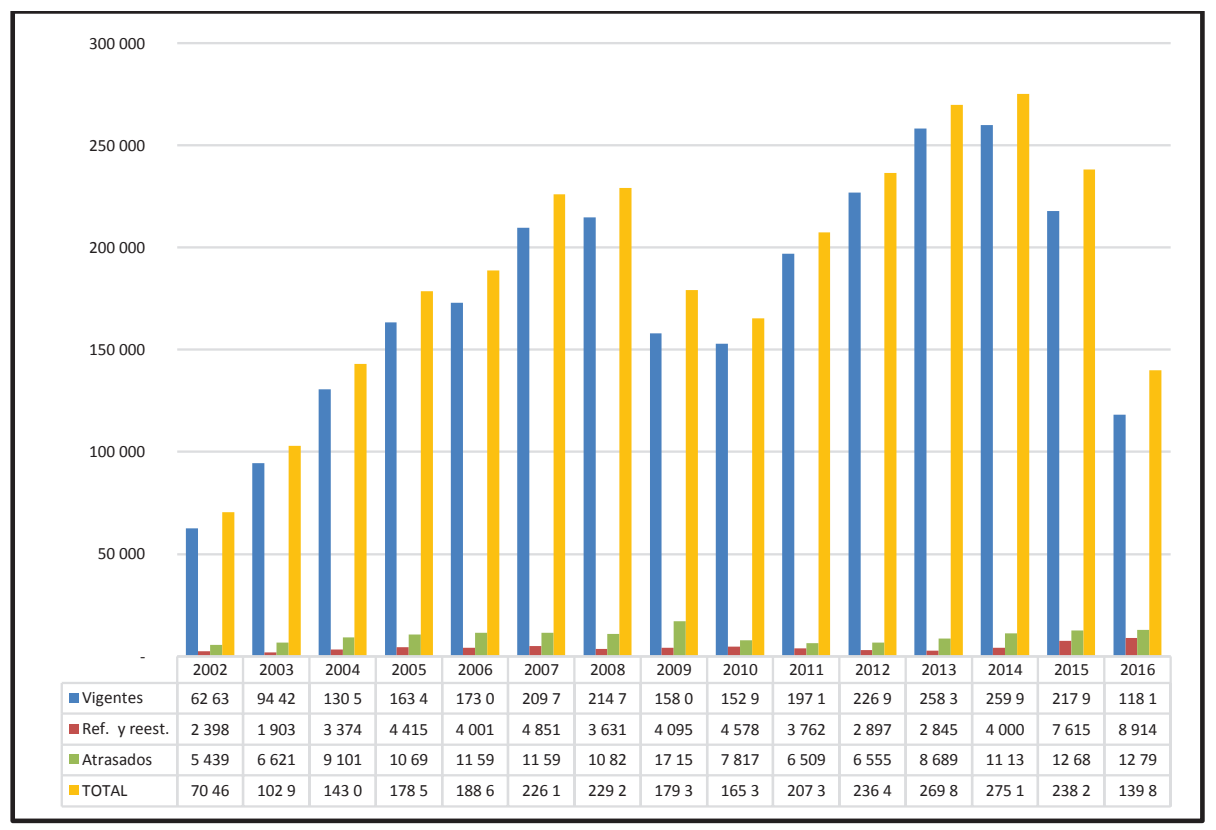

Figura 5. Promedio anual de los créditos vigentes, refinanciados -reestructurados y atrasados en ME de la CMAC Arequipa 2002-2016 (en miles de soles)

Fuente: INDICADORES FINANCIEROS. SBS, 2002-2016

Elaboración propia

Luego con el crecimiento se introducen exitosamente los créditos orientados a la Micro y Pequeña Empresa (MES) posteriormente llamados MYPES. La institución está comprometida en ofrecer mejores productos y servicios a las micro y pequeñas empresas. Los créditos comerciales cuyo monto debe ser superior a los US\$30,000.

Los créditos de consumo están compuestos por los créditos con descuentos por planilla del sector privado o público y los créditos personales. Ya en el año 2004, se puso a disposición de los clientes el Crédito Hipotecario para la adquisición o remodelación de la casa.

En el año 2004 los créditos de consumo de las MYPES han bajado respecto al 2003 por políticas internas.

La crisis financiera iniciada en EEUU, muestra una mayor intensidad en nuestra economía a mediados de 2008. El PBI no se ve afectado 
por influencia de la demanda interna sobre todo del sector no primario que fue superior al primario.

Se muestra una mayor demanda de los créditos de la pequeña empresa que es superior a la micro empresa y en esos sectores se ubica la mayor cantidad de clientes. Lo que ha conllevado que al 2014, la morosidad de la cartera de clientes se ha acentuado en el segmento empresarial: Pequeña, micro y mediana empresa. Por otro lado, la cartera de consumo e hipotecario mantienen niveles más estables a partir del 2011.

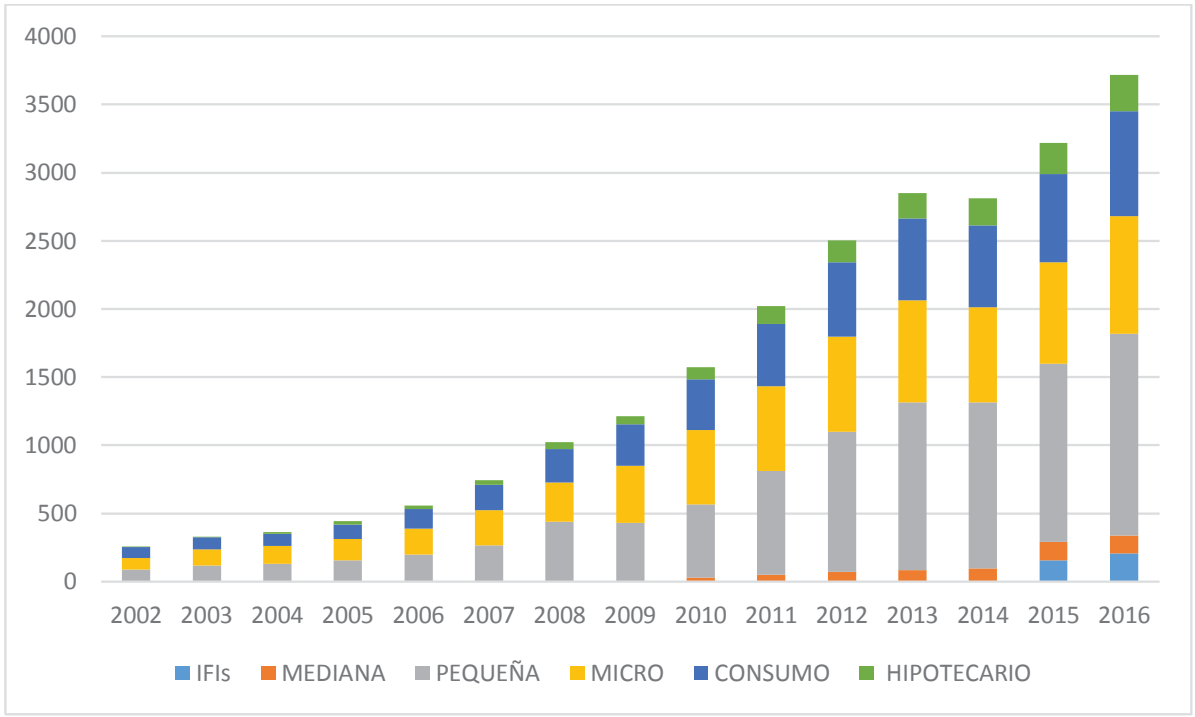

Figura 6. Créditos por sectores de la CMAC Arequipa 2002-2016 (en miles de soles) Fuente: Memorias anuales CMAC Arequipa, 2002-2016

Elaboración propia

\subsection{Análisis del ROA y el ROE}

Al final del 2016, la CMAC Arequipa logra mantener los indicadores de rentabilidad por encima del promedio de su sistema.

De hecho, que en todo el período en estudio el ROE es mayor al ROA; porque el segundo posee un mayor denominador por influencia del apalancamiento financiero de los depósitos de los clientes. 
La utilidad neta total tiene una caída entre el 2013 y 2014, sobre todo por las utilidades netas que en ME se hacen negativas.

En la Figura 7, se observa en el ROE una tendencia declinante sobre todo desde el 2006 y termina al 2016 a casi el 50\% del año 2002, el ROA disminuye levemente al 2016 respecto al período en estudio. En el 2013 cambian de software: Bantotal reemplaza a Sorfy, para tratar de detectar la mora oportunamente.

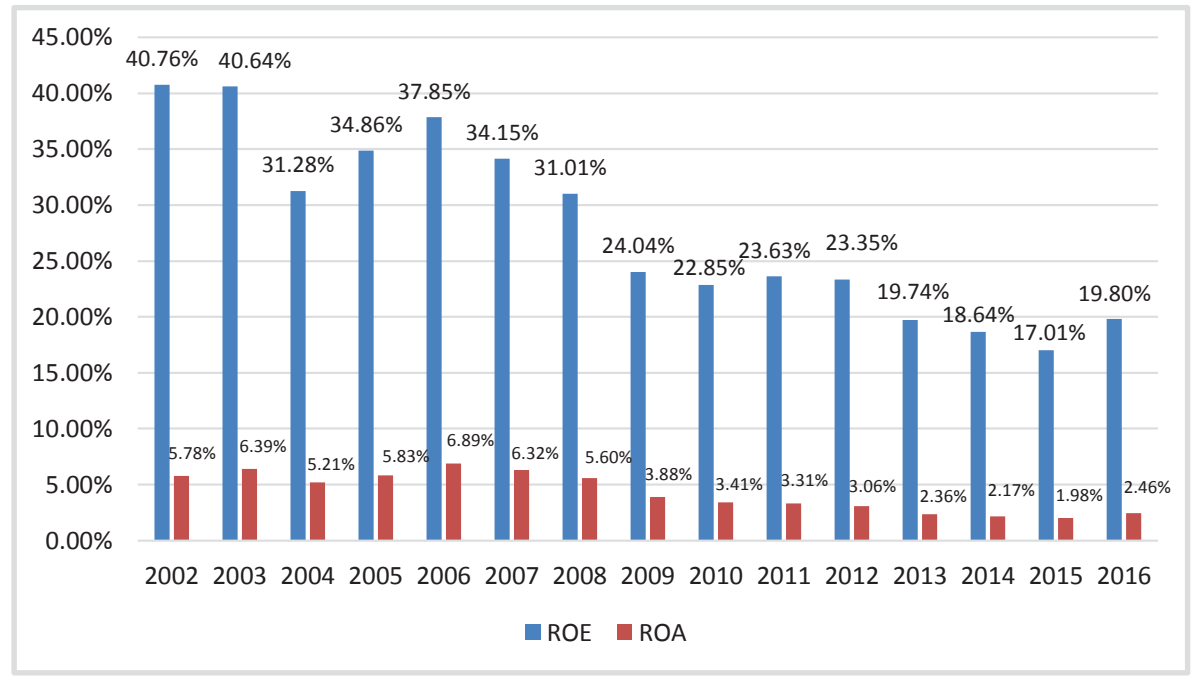

Figura 7. Evolución del ROE y ROA de la CMAC Arequipa (en \%). Período 2002-2016. Fuente: Indicadores Financieros. SBS, 2002-2016

Elaboración propia

\subsection{Correlación del ROA vs créditos directos, Inf. y el PBI real.}

La Figura 8, afirma la tendencia ascendente de los créditos directos totales, sin embargo, se presenta una correlación inversa respecto al ROA en la CMAC Arequipa, dado que esta última no va en la misma dirección de la primera, inclusive presentan una correlación alta de: 0.8389 de coeficiente de Pearson, durante el período del 2002-2016.

Así mismo, en la Figura 9, se presenta una correlación inversa muy baja respecto al ROA en la CMAC Arequipa de la inflación, de: 0.0239 de 
coeficiente de Pearson, tampoco van en la misma dirección durante el período del 2002-2016.

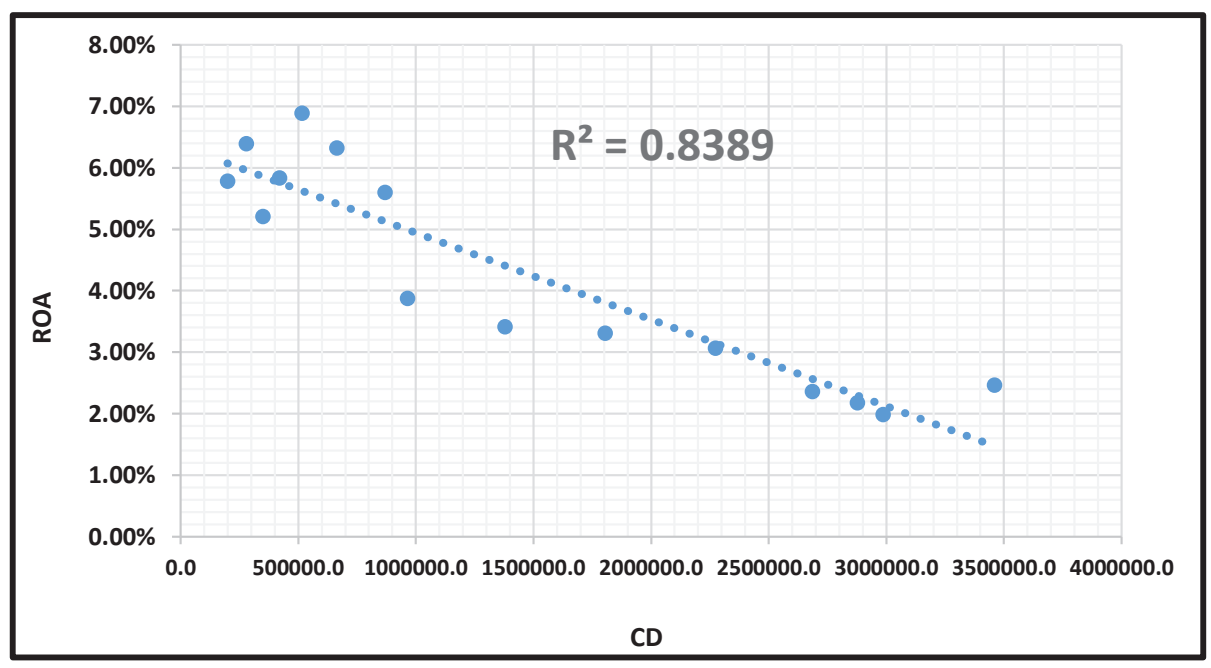

Figura 8. Correlación del ROA vs Créditos directos.

Fuente: Indicadores Financieros. SBS, 2002-2016.

Elaboración propia

Finalmente, en la Figura 10, la correlación es inversa alta respecto al ROA en la CMAC Arequipa del PBI real de: 0.8279 de coeficiente de Pearson, durante el período del 2002-2016. En este gráfico estadísticamente se está considerando los valores reales del PBI real.

\subsection{Correlación del ROE vs créditos directos, Inf. y el PBI real.}

La Figura 11, expresa la tendencia ascendente de los créditos directos totales, sin embargo, se presenta una correlación inversa respecto al ROE en la CMAC Arequipa, dado que esta última no ha va en la misma dirección de la primera. Inclusive presentan una correlación alta de: 0.801 de coeficiente de Pearson, durante el período del 2002-2016.

Así mismo, en la Figura 12, se presenta una correlación inversa muy baja respecto al ROE en la CMAC Arequipa de la inflación, de: 0.0534 de coeficiente de Pearson, durante el período del 2002-2016. 
Pensamiento Crítico Vol. 25. $\mathbf{N}^{\circ} 1$

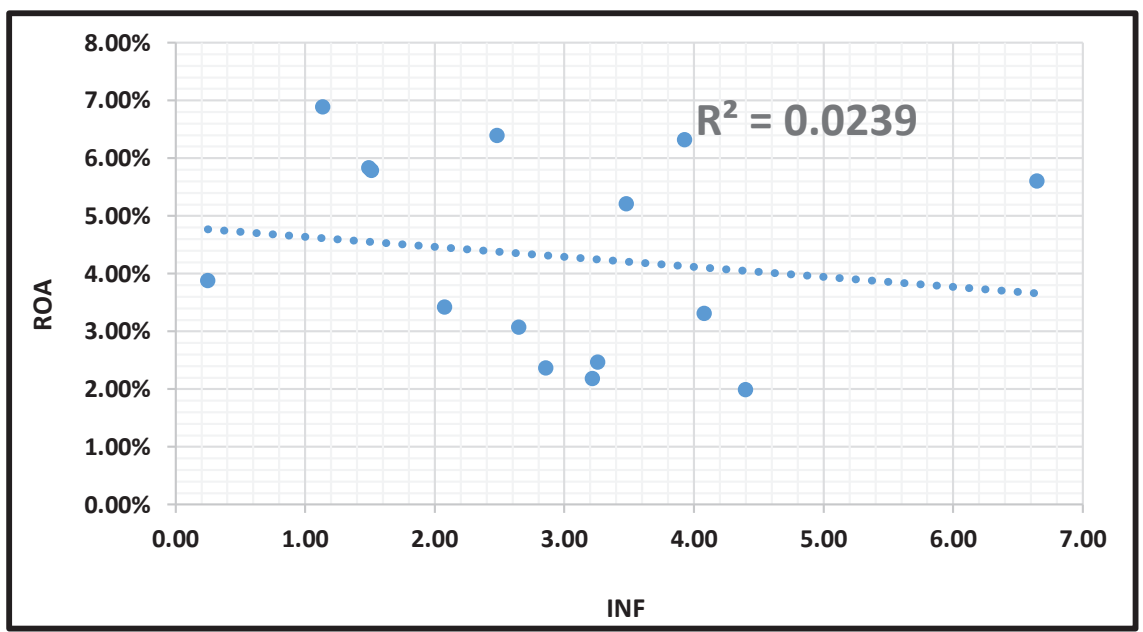

Figura 9. Correlación del ROA vs Inf.

Fuente: Indicadores Financieros. SBS, 2002-2016

Elaboración propia

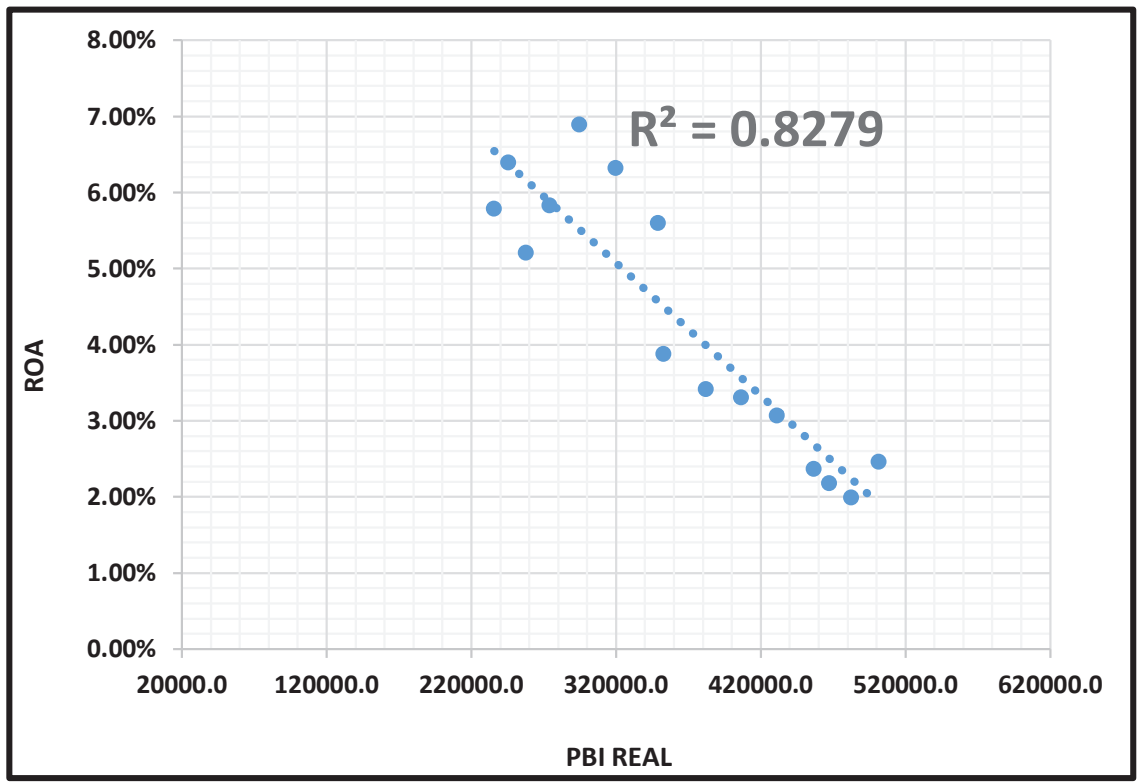

Figura 10. Correlación del ROA vs PBI real.

Fuente: Indicadores Financieros. SBS, 2002-2016

Elaboración propia 
Finalmente, en la Figura 13, la correlación es inversa alta respecto al ROE en la CMAC Arequipa con el PBI real de: 0.8733 de coeficiente de Pearson, durante el período del 2002-2016. En esta figura estadísticamente se está considerando los valores reales del PBI real.

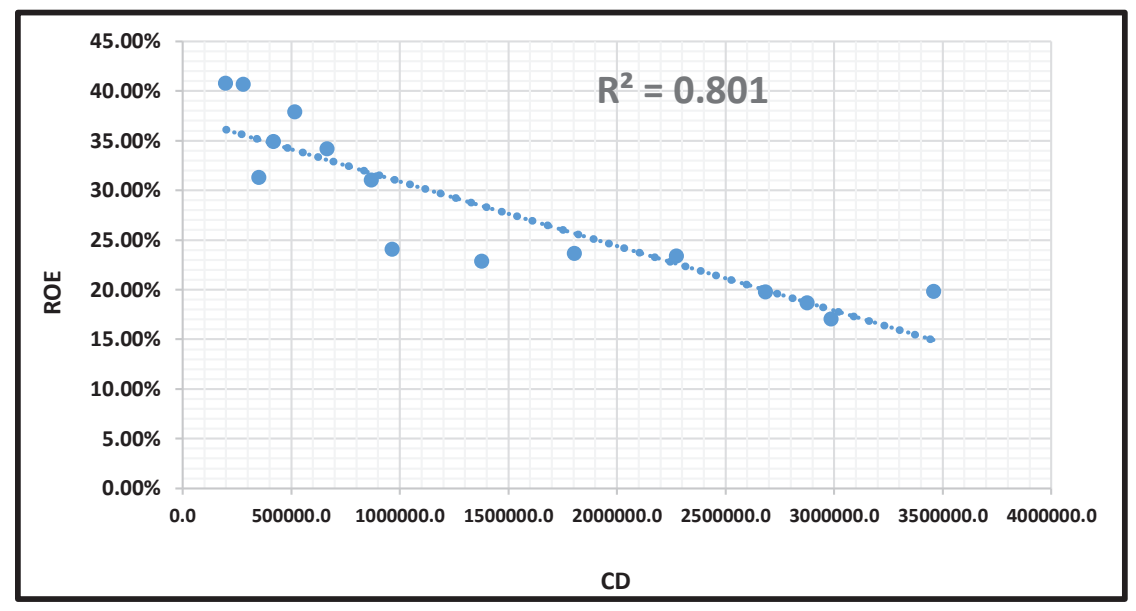

Figura 11. Correlación del ROE vs Créditos directos.

Fuente: Indicadores Financieros. SBS, 2002-2016

Elaboración propia

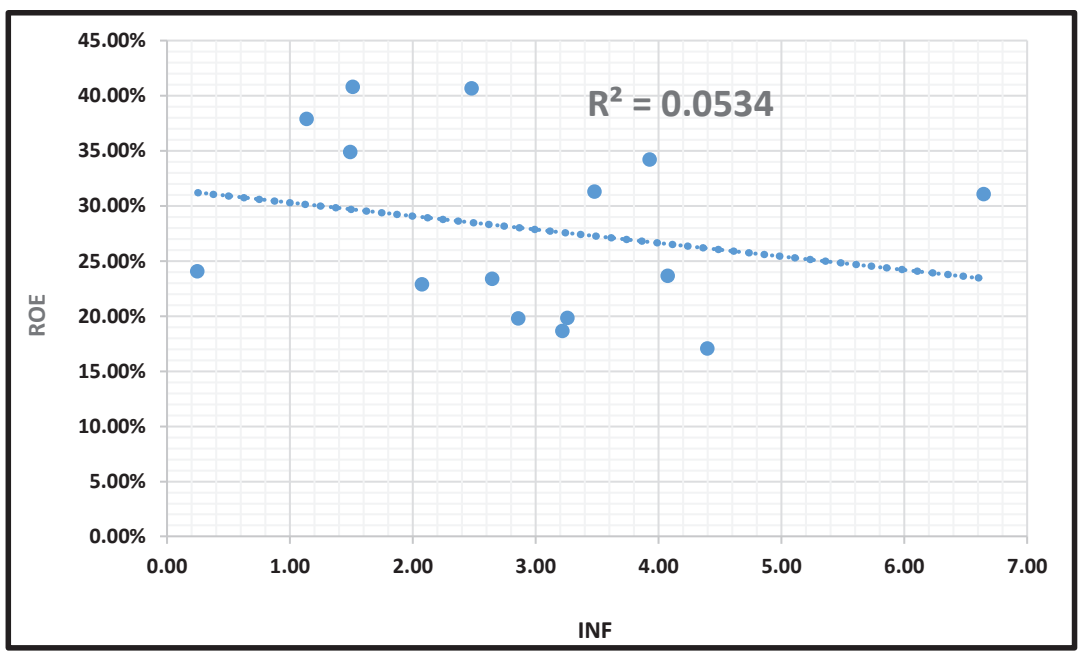

Figura 12. Correlación del ROE vs Inf.

Fuente: Indicadores Financieros. SBS, 2002-2016

Elaboración propia 


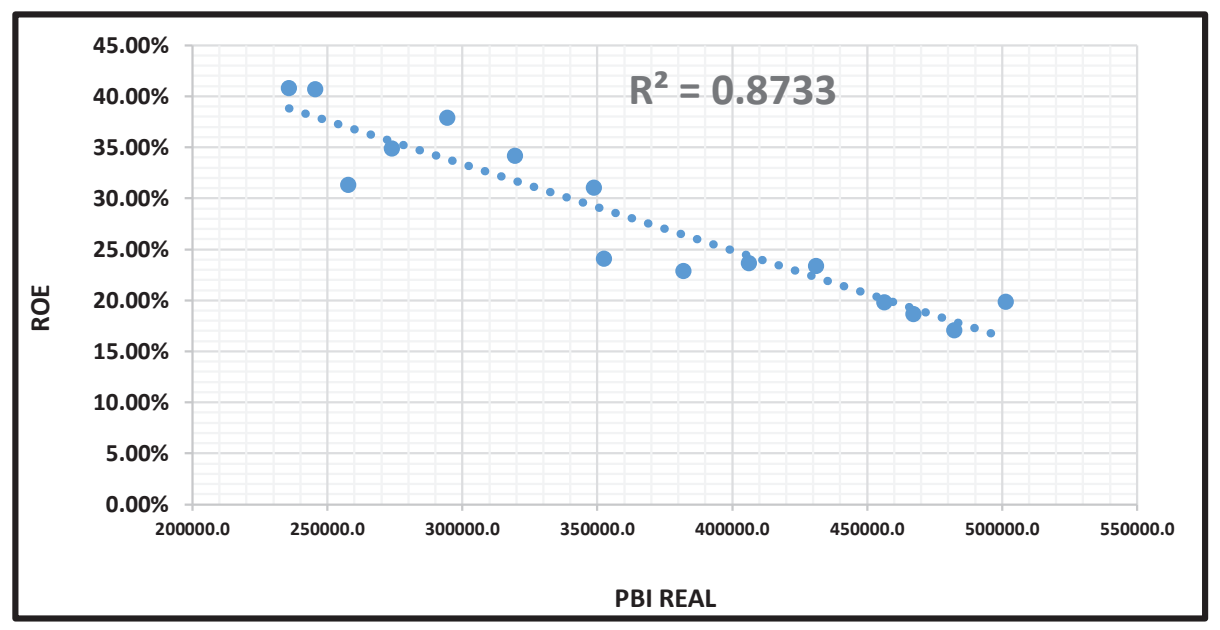

Figura 13. Correlación del ROE vs PBI real.

Fuente: Indicadores Financieros. SBS, 2002-2016

Elaboración propia

\subsection{Estimaciones para el modelo econométrico del ROA}

Este trabajo de investigación a partir de los resultados empíricos, pretende contribuir al entendimiento y evolución de ciertas variables financieras-económicas consideradas relevantes y que explican en cierta manera la rentabilidad de la CMAC Arequipa en el período en estudio.

Para el procesamiento de los datos se usó el software Econometric Views, se corrieron dos modelos econométricos de Mínimos cuadrados ordinarios para medir la relación de las variables, luego para corregir las violaciones de supuesto se realizaron modelos autorregresivos y con aplicación de promedios móviles específicamente modelos ARMA, se obtuvo la adecuada relación de las variables cuantitativas sin perturbaciones

Por lo tanto, en la tabla 2 , se muestran los resultados de las estimaciones para el ROA. se aprecia que el modelo tiene un indicador de autocorrelación parcial con violación del supuesto de mínima autocorrelación, de acuerdo a la prueba de Correlograma del Q estadístico para el ROA cuyos resultados recomiendan para levantar el supuesto aplicar al modelo un rezago de dimensión 1 y asimismo un promedio móvil de dimensión 7 , se aprecia que el modelo tiene un valor de la prueba de Jarque-Bera para 
el ROA cuyo coeficiente es 0.565073 y una probabilidad del 0.753869 , que ante un nivel de confianza del 95\%, se puede afirmar que el modelo presenta normalidad. Se tiene un AR(1) con un coeficiente de 0.375636 la cual es menor a 1 absoluto lo que da la condición de estacionario. Los créditos directos tienen una correlación inversa y es significativa en 0.0480, la inflación tiene una correlación directa pero no es significativa en 0.8739 y la variación del PBI real como variable dummy tiene una correlación directa pero no es significativa en 0.7128. El Prob (F-statistic) con un valor de 0.000253 que es menor al valor de probabilidad del 0.05 por lo que indica que el modelo es significativo y un Durbin-Watson que se aproxima a 2 con un valor de 1.822353 con el cual se corrige la presencia de autocorrelación, según Gujarati, D. y Porter, D. (2010, p. 413) significa "como la correlación entre miembros de series de observaciones ordenadas en el tiempo".

Tabla 2

Resultados de las estimaciones para el ROA

\begin{tabular}{|l|l|l|l|}
\hline \multicolumn{2}{|l|}{ Variable dependiente. Retorno sobre el activo (ROA) } & Std. Error & P-value \\
\hline Variable & Coeficiente & 0,468796 & 0,4461 \\
\hline AR(1) & 0,375636 & 5,20 E- 07 & 0,0480 \\
\hline CD & $-1,21 E-06$ & 0,100685 & 0,8739 \\
\hline INF & 0,016494 & 0,446067 & 0,7128 \\
\hline PBIR_DUMMY & 0,170181 & & 0,000253 \\
\hline Prob(F-statistic) & & & 1,822353 \\
\hline Durbin- Watson & & & 0,565073 \\
\hline Prueba de Jarque-Bera & Coeficiente & & 0,753869 \\
\hline & Probabilidad & & 15 años \\
\hline Numero de observaciones & & & \\
\hline
\end{tabular}

Fuente: Indicadores Financieros. SBS, 2002-2016

Elaboración propia

\subsection{Estimaciones para el modelo econométrico del ROE}

Enseguida, se presenta la tabla 3, y de los resultados de las estimaciones para el ROE, se aprecia que el modelo tiene un indicador de autocorrelación parcial con violación del supuesto de mínima autocorrelación, de 
acuerdo a la prueba de Correlograma del Q estadístico para el ROE cuyos resultados recomiendan para levantar el supuesto aplicar al modelo un rezago de dimensión 1 y asimismo un promedio móvil de dimensión 7 , se aprecia que el modelo tiene un valor de la prueba de Jarque-Bera para el ROE cuyo coeficiente es 0.357644 y una probabilidad del 0.836255 , que ante un nivel de confianza del 95\%, se puede afirmar que el modelo presenta normalidad. Se tiene un AR(1) con un coeficiente de 0.250277 la cual es menor a 1 absoluto lo que da la condición de estacionario Los créditos directos tienen una correlación inversa y es significativa en 0.0096, la inflación tiene una correlación inversa pero no es significativa en 0.4701 y la variación del PBI real como variable dummy tiene una correlación directa pero no es significativa en 0.6694. El Prob (F-statistic) con un valor de 0.000425 que es menor al valor de probabilidad del 0.05 por lo que indica que el modelo es significativo y un Durbin-Watson que se aproxima a 2 con un valor de 1.712736 con el cual estamos corrigiendo la presencia de autocorrelación, según Gujarati, D. y Porter, D. (2010, p. 413) significa "como la correlación entre miembros de series de observaciones ordenadas en el tiempo".

Tabla 3

Resultados de las estimaciones para el ROE

\begin{tabular}{|l|l|l|l|}
\hline \multicolumn{4}{|l|}{ Variable dependiente. Retorno sobre el patrimonio (ROE) } \\
\hline Variable & Coeficiente & Std. Error & P-value \\
\hline AR(1) & 0,250277 & 0,319115 & 0,4555 \\
\hline CD & $-5,78 \mathrm{E}-06$ & $1,71 \mathrm{E}-06$ & 0,0096 \\
\hline INF & $-0,436457$ & 0,575671 & 0,4701 \\
\hline PBIR_DUMMY & 0,785790 & 1,773179 & 0,6694 \\
\hline Prob(F-statistic) & & & 0,000425 \\
\hline Durbin-Watson & & & 1,712736 \\
\hline Prueba de Jarque-Bera & Coeficiente & & 0,357644 \\
\hline & Probabilidad & & 0,836255 \\
\hline Numero de observaciones & & & 15 años \\
\hline
\end{tabular}

Fuente: Indicadores Financieros. SBS, 2002-2016

Elaboración propia 
El modelo econométrico del ROA es el que se ajusta mejor según lo mostrado empíricamente, por tener un más alto valor de Durbin-Watson cercano al 2 lo que indica una correlación serial positiva.

\section{Conclusiones}

En el aspecto financiero, la CMAC Arequipa, cuenta con un crecimiento ascendente de los créditos directos en MN (figura 4) y con un crecimiento fluctuante de los créditos directos en ME con tendencia descendente (figura 5). Lo positivo es que se ha logrado una expansión microcrediticia favoreciendo fundamentalmente a MYPES y al consumo de los hogares con bajos ingresos. Sin embargo, el aporte neto de los créditos directos no contribuye en la rentabilidad de la CMAC Arequipa en el período en estudio porque se observa una tendencia descendente del ROA y del ROE (figura 7).

La creación de las cajas municipales de ahorro y crédito y bajo la supervisión de una entidad como la Superintendencia de Banca y Seguros permite regular el desenvolvimiento de estas y la gestión del dinero del público.

Los créditos directos de la CMAC Arequipa desde el 2002 se han orientado a las MYPES, especialmente del sector comercio, transporte e Inmobiliario (figura 6).

Hay que mencionar que asumir un riesgo es algo inherente al ejercicio financiero y a partir del 2008 se observa una tendencia descendente de las rentabilidades. El Bantotal es un software de información empresarial que permite procesar los datos de un negocio sobre todo a nivel corporativo.

Existe una Correlación significativamente inversa para el ROA y el ROE en relación a la colocación créditos directos. Ello es producto de la disyuntiva que enfrenta la CMAC Arequipa, puesto que trata de mejorar su rentabilidad colocando mayor cantidad de créditos a personas naturales y jurídicas con buena calificación crediticia. Sin embargo, estos créditos en su mayoría son dados al sector informal MYPE, debido a que la institución tiene una misión de inclusión social financiera, por lo cual otorga créditos a clientes con mayor nivel de riesgo crediticio, afectando ello de manera negativa su rentabilidad. Lo mencionado anteriormente impli- 
ca que la mayor colocación de los créditos directos por parte de CMAC Arequipa tendrá una implicancia negativa en el ROA y el ROE, por el hecho de que dicha variable también depende del nivel de morosidad de la cartera de la institución. Observándose que existe una tendencia creciente de la morosidad en el período analizado, a su vez esto implica una mayor cantidad de provisiones de dichos créditos, por lo que vendría afectando la rentabilidad de la CMAC Arequipa.

Hay que considerar que la inflación es una variable exógena y con un tipo de riesgo externo a la gestión de la empresa. El aumento de la inflación implica una mayor posibilidad de no pago por los clientes de la CMAC Arequipa, pues su poder adquisitivo disminuye.

Las fluctuaciones de la variación del PBI real, variable independiente, expresa la producción de los diferentes sectores que afectan las condiciones del mercado en: favorables o desfavorables para el sector financiero e inciden en el ROA y el ROE de las instituciones.

La relación del sistema financiero con las tecnologías de la información ha reducido los riesgos, siendo la innovación uno de los grandes pilares para el desarrollo de estas.

\section{Referencias Bibliográficas}

\section{Revistas:}

Aguilar, G. (2013). Microfinanzas y Crecimiento regional en el Perú. Pontificia Universidad Católica del Perú. Publicado el 27 de septiembre del 2013. p. 143-173.

Banco Central de Reserva del Perú. BCRP. (2017). 20 años con inflación anualizada de un dígito. http://www.bcrp.gob.pe/docs/Transparencia/Notas-Informativas/2017/nota-informativa-2017-02-07.pdf. Publicado el 07 de febrero del 2017. $2 \mathrm{p}$.

Castilla, A. (2015). Proyecto LINK y Econometría de Alta Frecuencia: Las últimas aportaciones econométricas de Lawrence R. Klein. Estudios de Economía Aplicada; Madrid. Vol. 33, No 2, p. 421-450.

Conde, C. (2012). Diversificación del riesgo en las instituciones microfinancieras. México. Universidad Autónoma de Metropolitana Xochimilco. p. 81-99. 
Duflo, E., Banerje, A., Glennerster, R. y Kinnan, C. (2013). The miracle of microfinance? Evidence from a randomized evaluation. National bureau of economic research. http://www.nber.org/papers/w18950. p. 1-37.

Instituto Nacional de Estadística e Informática-INEI. Metodología de Cálculo del Producto Bruto Interno Anual-INEI, https://www.inei.gob.pe/media/MenuRecursivo/metodologias/pbi02.pdf. 48p.

Jaramillo, M. (2014). El modelo de la caja Municipal en Perú-Grade. https://www.grade.org.pe/publicaciones/13151-el-modelo-de-la-caja-municipal-en-peru/. 9p.

Markowitz, J. (1952). Portfolio Selection. The Journal of Finance, Vol. 7, No. 1, p. 77-91. https://www.jstor.org/stable/2975974?seq=1

Puertas, R. y Marti, M. (2012). Análisis del Credit Scoring. RAE. DOI: 10.1590/S0034-75902013000300007.

Superintendencia de Banca, Seguros y AFP. (2017). https://www.sbs.gob.pe/ app/stats_net/stats/EstadisticaBoletinEstadistico.aspx?p=3\#.

\section{Libros:}

Gujarati, D. y Porter, D. (2010). Econometría. (5a edición). México, D.F: Mc GrawHill/Interamericana editores, S.A. de C.V. 921p.

Keynes, J. (1981). Teoría General de la ocupación, el interés y el dinero. (1 $1^{a}$ Edición en español). (Edición original 1943). México: Fondo de Cultura Económica. 337p.

Ross, S.; Westerfield, R. y Jordan, B. (2014). Fundamentos de Finanzas Corporativas. (Décima Edición). México, D.F: Mc Graw-Hill/Interamericana editores, S.A. de C.V. 991p.

Schumpeter, J. (1976). Teoría del desenvolvimiento económico. ( $1^{a}$ Edición en español). (Edición original 1944). México: Fondo de Cultura Económica. 255p.

\section{Tesis:}

Bayona, F. (2013). Análisis de los Factores que influyen en la rentabilidad de las cajas municipales de Ahorro y crédito en el Perú. Tesis de pregrado en Economía, Universidad de Piura, Perú, 107p.

\section{Leyes, sentencias y decretos:}

Presidencia de la República. (1980). Decreto Ley 23039 de mayo 14 de 1980. Norma el funcionamiento de las Cajas municipales de Ahorro y Crédito del Perú. 2p. 
Presidencia de la República (1990). Decreto Supremo 157-90-EF de mayo 29 de 1990. Norma funcionamiento en el país de las Cajas Municipales de Ahorro y Crédito. 9p.

Presidencia de la República (2008). Decreto Supremo 007-2008-TR de setiembre 30 del 2008. Texto único ordenado de la ley de promoción de la competitividad, formalización y desarrollo de la empresa y del acceso al empleo decente, ley MYPE. 12p.

\section{Institucional:}

Caja Arequipa. Memorias anuales 2002-2016. Recuperado de https://www.cajaarequipa.pe

\section{Entrevista:}

Espejo, Elvis (10 de Julio de 2018). Gerente de Agencia Caja Arequipa-Agencia Alfredo Mendiola. Los Olivos. Lima. Perú.

\section{Notas al final}

1 El articulo manifiesta un modelo econométrico de variables: financieras (créditos directos) y macroeconómicas (inflación y la variación del PBI real expresada como una variable dummy) y el tipo de artículo es de reflexión.

2 Economista, Facultad de Ciencias Económicas, Universidad Nacional Mayor de San Marcos, Lima, Perú. Calle Germán Amezaga 375, Lima, Perú. E-mail: edwin.bazan@unmsm.edu.pe. ORCID: 0000-0002-4415-6539 
